\title{
Development of Tdp1 inhibitors based on natural biologically active compounds
}

\author{
A.A. Chepanova ${ }^{1 *}$, A.L. Zakharenko ${ }^{1}$, O.D. Zakharova ${ }^{1}$, T.M. Khomenko ${ }^{2}$, \\ E.V. Suslov ${ }^{2}$, N.S. Li-Zhulanov ${ }^{2}$, K.P. Volcho ${ }^{2}$, O.I. Lavrik ${ }^{1}$ \\ ${ }^{1}$ Institute of Chemical Biology and Fundamental Medicine SB RAS, Novosibirsk, Russia \\ ${ }^{2}$ Institute of Organic Chemistry SB RAS, Novosibirsk, Russia \\ *e-mail: arinachepanova@mail.ru
}

Key words: tyrosyl-DNAphosphodiesterase I, inhibitor of tyrosyl-DNA phosphodiesterase I

Motivation and Aim: Tyrosyl-DNA phosphodiesterase 1 (Tdp1) is a promising target for anticancer therapy based on damage of tumor DNA caused by topoisomerase-1 (Top1) inhibitors. Tdp1 plays a key role in the repair of Top1-DNA covalent complexes formed by Top 1 inhibitors such as camptothecin and its clinical derivatives.

Presumably, inhibition of Tdp1 can enhance the therapeutic effect of Tor1 inhibitors, sensitizing tumor cells to their action [1].

The development of new inhibitors of DNA repair enzymes based on natural compounds and their derivatives is particularly relevant, since such compounds often have complementarity to targets of biological origin. We used derivatives of coumarin, terpenes and chromen for Tdp1 inhibitors screening.

Methods and Algorithms: Screening of compounds - potential inhibitors is carried out using a real-time fluorescence measurement method, which makes it possible to determine the initial reaction rate with high accuracy [2]. The effect of the selected compounds on the proliferation of transplanted tumor cell lines and the evaluation of cell death are studied using the MTT test.

Results: All studied compounds have a pronounced inhibitory effect. The dependence of the inhibitory activity on the structure of the compounds was revealed. Among the developed inhibitors, there are both moderately toxic and non-toxic compounds.

Conclusion: The compounds studied can be used to develop on their basis more effective inhibitors of Tdp1. The therapeutic effect of such substances can be a selective increase in the activity of Top1 inhibitors in tumors. Non-toxic inhibitors of Tdp1 are of particular interest, since will avoid additional side effects.

Asknowledgments: Supported by Russian Science Foundation (grant No. 16-13-10074).

\section{References}

1. Dexheimer T.S. et al. (2008) Anticancer Agents Med. Chem. 8:381-389.

2. Zakharenko et al. (2015) Bioorg. Med. Chem. 9:2044-2052. 